\title{
SLFN14 mutations underlie thrombocytopenia with excessive bleeding and platelet secretion defects
}

\author{
Sarah J. Fletcher, ${ }^{1}$ Ben Johnson, ${ }^{1}$ Gillian C. Lowe, ${ }^{1}$ Danai Bem, ${ }^{1}$ Sian Drake, ${ }^{1}$ Marie Lordkipanidzé, ${ }^{1}$ Isabel Sánchez Guiú, ${ }^{1,2}$ \\ Ban Dawood, ${ }^{1}$ José Rivera, ${ }^{2}$ Michael A. Simpson, ${ }^{3}$ Martina E. Daly, ${ }^{4}$ Jayashree Motwani, ${ }^{5}$ Peter W. Collins, ${ }^{6}$ Steve P. Watson, ${ }^{1}$ \\ and Neil V. Morgan ${ }^{1}$ on behalf of the UK Genotyping and Phenotyping of Platelets study group

\begin{abstract}
'Centre for Cardiovascular Sciences, School of Clinical and Experimental Medicine, College of Medical and Dental Sciences, University of Birmingham, Birmingham, United Kingdom. ${ }^{2}$ Centro Regional de Hemodonación, Universidad de Murcia, IMIB-Arrixaca, Murcia, Spain. ${ }^{3}$ Division of Cenetics and Molecular Medicine, King's College, London, United Kingdom. ' ${ }^{4}$ Department of Cardiovascular Science, University of Sheffield Medical School, University of Sheffield, Sheffield, United Kingdom. ${ }^{5}$ Department of Haematology, Birmingham Children's Hospital, Birmingham, United Kingdom. ${ }^{6}$ Arthur Bloom Haemophilia Centre, School of Medicine, Cardiff University, Cardiff, United Kingdom.
\end{abstract}

\begin{abstract}
Inherited thrombocytopenias are a group of disorders that are characterized by a low platelet count and are sometimes associated with excessive bleeding that ranges from mild to severe. We evaluated 36 unrelated patients and 17 family members displaying thrombocytopenia that were recruited to the UK Genotyping and Phenotyping of Platelets (GAPP) study. All patients had a history of excessive bleeding of unknown etiology. We performed platelet phenotyping and whole-exome sequencing (WES) on all patients and identified mutations in schlafen 14 (SLFN14) in 12 patients from 3 unrelated families. Patients harboring SLFN14 mutations displayed an analogous phenotype that consisted of moderate thrombocytopenia, enlarged platelets, decreased ATP secretion, and a dominant inheritance pattern. Three heterozygous missense mutations were identified in affected family members and predicted to encode substitutions (K218E, K219N, and V220D) within an ATPase-AAA-4, GTP/ATP-binding region of SLFN14. Endogenous SLFN14 expression was reduced in platelets from all patients, and mutant SLFN14 expression was markedly decreased compared with that of WT SLFN14 when overexpressed in transfected cells. Electron microscopy revealed a reduced number of dense granules in affected patients platelets, correlating with a decreased ATP secretion observed in lumiaggregometry studies. These results identify SLFN14 mutations as cause for an inherited thrombocytopenia with excessive bleeding, outlining a fundamental role for SLFN14 in platelet formation and function.
\end{abstract}

\section{Introduction}

Inherited thrombocytopenias are a group of disorders associated with bleeding of varying severity, depending both on the reduction in platelet count and any additional platelet dysfunction (1). The normal human platelet count ranges widely $\left(150 \times 10^{9}\right.$ to $400 \times 10^{9}$ platelets/l) and is maintained within a narrow range for each individual. This homeostasis requires a balance among thrombopoiesis, platelet senescence, and consumption. Heritable forms of thrombocytopenia are usually caused by mutations in genes involved in platelet production and megakaryocytic differentiation. Over 20 forms of inherited thrombocytopenia have been described in the OMIM database (http://www.ncbi.nlm.nih.gov/omim); however, in approximately $50 \%$ of patients, the causative gene remains unknown $(2,3)$. Identification of such genes is fundamental to providing information on proteins involved in normal platelet physiology and is critical for developing our understanding of disease pathogenesis.

To date, the UK Genotyping and Phenotyping of Platelets (GAPP) study (4) has investigated over 500 patients with excessive bleeding of unknown etiology, identifying a platelet defect in approximately $60 \%$ of participants. A key criterion for recruitment

Conflict of interest: The authors have declared that no conflict of interest exists. Submitted: January 13, 2015; Accepted: July 8, 2015.

Reference information: J Clin Invest. 2015;125(9):3600-3605. doi:10.1172/JCI80347. is that known genetic causes of platelet dysfunction have been ruled out on the basis of functional studies and targeted gene sequencing. Platelets from patients recruited to this study undergo extensive phenotypic analysis, including lumiaggregometry on a variety of platelet agonists and detailed analysis of platelet number and morphology. This is followed by whole-exome sequencing (WES), which has greatly enhanced the probability and speed of identification of novel causative gene mutations in such conditions (5-7). In this study, we demonstrate how combined phenotyping and genotyping has enabled us to identify 3 single nucleotide variations in a gene that we believe to be novel, schlafen 14 (SLFN14), predicting substitutions of 3 consecutive amino acids in SLFN14, in 3 unrelated families displaying a moderate form of congenital thrombocytopenia and a strong bleeding history. These individuals had a more severe bleeding history than predicted on platelet count alone, with platelets displaying reduced aggregation and ATP secretion in response to several platelet agonists, including ADP, collagen, and protease-activated receptor-1-activating (PAR-1-activating) peptide. This phenotyping and genotyping approach has identified SLFN14 as causative gene for a form of thrombocytopenia.

\section{Results and Discussion}

There are approximately 3,000 patients with platelet function disorders associated with excessive bleeding within the UK Hae- 
Table 1. Heterozygous SLFN14 mutations and Platelet Phenotyping in affected patients of the 3 families with thrombocytopenia, excessive bleeding and platelet secretion defects

\begin{tabular}{|c|c|c|c|c|c|c|c|}
\hline Family/patient ID & $\begin{array}{c}\text { SLFN14 } \\
\text { nucleotide } \\
\text { alteration }{ }^{\mathrm{A}}\end{array}$ & $\begin{array}{l}\text { Effect on } \\
\text { SLFN14 } \\
\text { protein }\end{array}$ & $\begin{array}{l}\text { Platelet } \\
\text { count } \\
\left(\times 10^{9} / /\right)^{\mathrm{B}}\end{array}$ & $\begin{array}{l}\text { Mean platelet } \\
\text { volume (fl) }\end{array}$ & ISTH BAT score ${ }^{\complement}$ & $\begin{array}{l}\text { Lumiaggregometry/flow } \\
\text { cytometry defects }\end{array}$ & $\begin{array}{c}\text { ATP secretion } \\
\left(\mathrm{nmol} / 1 \times 10^{8} \text { platelets }\right)^{\mathrm{D}}\end{array}$ \\
\hline A; I:1 & c. $659 \mathrm{~T}>\mathrm{A}$ & p.Val220Asp & NA & NA & NA & NA & NA \\
\hline$A ; \| I: 2$ & c.659 T>A & p.Val220Asp & 140 & 9.1 & 5 & $\begin{array}{l}\operatorname{ADP}(10,30,100 \mu \mathrm{M}) \text {, collagen } \\
(1,3 \mu \mathrm{g} / \mathrm{ml}), \text { PAR-1 }(100 \mu \mathrm{M})\end{array}$ & 0.81 \\
\hline A; III:3 & c.659 T>A & p.Val220Asp & 74 & 10.4 & 10 & $\begin{array}{c}\text { ADP }(10 \mu \mathrm{M}) \text {, collagen }(3 \mu \mathrm{g} / \mathrm{ml}) \text {, } \\
\text { PAR-1 }(30 \mu \mathrm{M})\end{array}$ & 0.42 \\
\hline $\mathrm{A} ; \mathrm{IV}: 2$ & c.659 T>A & p.Val220Asp & 110 & 9.3 & 13 & $\begin{array}{c}\operatorname{ADP}(10,30 \mu \mathrm{M}), \text { collagen }(1,3 \mu \mathrm{g} / \mathrm{ml}) \text {, } \\
\text { PAR-1 }(100 \mu \mathrm{M})\end{array}$ & ND \\
\hline A; IV:4 & c. $659 \mathrm{~T}>\mathrm{A}$ & p.Val220Asp & 100 & 11.1 & 22 & $\begin{array}{c}\text { ADP }(30 \mu \mathrm{M}) \text {, collagen }(3 \mu \mathrm{g} / \mathrm{ml}) \text {, } \\
\text { PAR-1 }(100 \mu \mathrm{M})\end{array}$ & 0.28 \\
\hline$A ; I V: 5$ & c.659 T>A & p.Val220Asp & 116 & 11.2 & 21 & $\begin{array}{c}\text { ADP }(10 \mu \mathrm{M}) \text {, collagen }(3 \mu \mathrm{g} / \mathrm{ml}) \text {, } \\
\text { PAR-1 }(30 \mu \mathrm{M})\end{array}$ & 0.48 \\
\hline B; II:3 & c. $657 A>T$ & p.Lys219Asn & 68 & 11.9 & 20 & $\begin{array}{l}\text { Flow cytometry reduced responses } \\
\text { to high conc. CRP and PAR-1 }\end{array}$ & NA \\
\hline$B ; 1: 2$ & c.657 A>T & p.Lys219Asn & 83 & 11.9 & 13 & $\begin{array}{c}\text { ADP }(10,30 \mu \mathrm{M}) \text {, collagen } \\
(3,10 \mu \mathrm{g} / \mathrm{ml}), \text { PAR-1 }(100 \mu \mathrm{M})\end{array}$ & 0.63 \\
\hline C; II:2 & c. $652 A>G$ & p.Lys218Glu & 89 & 13.0 & NA & $\begin{array}{l}\operatorname{ADP}(10,100 \mu \mathrm{M}) \text {, collagen } \\
(1,3 \mu \mathrm{g} / \mathrm{ml}), \text { PAR-1 }(100 \mu \mathrm{M})\end{array}$ & 0.12 \\
\hline
\end{tabular}

Heterozygous nucleotide changes present in SLFN14 and their predicted effects on the resulting protein are shown. Index cases are shown in bold. ${ }^{A}$ Alterations are numbered according to positions in the NM_001129820. ${ }^{B}$ Mean platelet counts are shown. Normal reference range is $150 \times 10^{9}$ platelets $/ \mathrm{I}$ to $400 \times 10^{9}$ platelets/l. Thrombocytopenia is defined as platelet count $<150 \times 10^{9}$ platelets/l. For mean platelet volume, the reference range is $7.83-10.5 \mathrm{fl}$. International Society on Thrombosis and Haemostasis bleeding assessment tool (BAT) score. The 95th percentile (score of 4) was calculated from healthy volunteers (17). ${ }^{D}$ ATP secreted in response to $100 \mu \mathrm{M}$ PAR-1 receptor-specific peptide SFLLRN. The 5 th percentile in healthy volunteers is $0.82 \mathrm{nmol} / 1 \times 10^{8}$ platelets. conc., concentration; CRP, collagen-related peptide; NA, not available; ND, not detectable.

mophilia Comprehensive Care Centres, of which approximately $10 \%$ have a reduced platelet count. A candidate gene mutation has not been identified in approximately $50 \%$ of these patients. Over 500 patients from 25 UK Haemophilia Comprehensive Care Centres with excessive bleeding and suspected platelet function disorders have been recruited to the UK GAPP study. Of these patients, $13 \%$ have been classified as having a thrombocytopenia of unknown cause. A strong family history of low platelet count and significant bleeding phenotype enabled prioritization of patients for gene identification studies. WES was performed and analysis was undertaken in 53 patients ( 36 index cases) displaying reduced platelet counts ranging from $15 \times 10^{9} / 1$ to $140 \times 10^{9} / \mathrm{l}$. Over 3 generations, family A had 9 affected members with a strong bleeding history and moderate thrombocytopenia, with platelet counts ranging from $74 \times 10^{9} / 1$ to $140 \times 10^{9} / 1$ (Figure $1 \mathrm{~A}$ and Table 1 ). The proband, IV:4, was aged 31 years with a platelet count of $100 \times 10^{9} / 1$ when entering the study. Her history included frequent and severe cutaneous bruising, prolonged bleeding from minor wounds, menorrhagia, postpartum hemorrhage, and spontaneous muscle hematoma. Several other members of family A also had significant bleeding histories and moderate thrombocytopenia (Table 1).

The gold-standard test for platelet function is Born aggregometry, but increased information can be obtained by real-time measurement of secretion of ATP (lumiaggregometry) (8). We tested affected members of family A (III:2, III:3, IV:2, IV:4, and IV:5) and observed reduced aggregation to ADP $(10$ and $30 \mu \mathrm{M})$, collagen $(3 \mu \mathrm{g} / \mathrm{ml}$ and $10 \mu \mathrm{g} / \mathrm{ml})$, and PAR-1-activating peptide $(100 \mu \mathrm{M})$, with reduced ATP secretion (Figure 2, A and B). The similar platelet phenotype in the affected individuals of family A was consistent with a dominant mode of inheritance.

Exome sequencing of DNA from IV:2, IV:4, and III:3 in family A revealed 22,867, 23,334, and 23,153 sequence variations, respectively. Comparisons with dbSNP build 135, the 1000 Genomes project database, and our in-house database (composed of $>600$ exomes) identified 124, 137, and 128 heterozygous variants we believe to be novel, respectively. Only 8 variants were shared by the 3 affected patients, of which 4 resulted in amino acid changes, including 3 nonsynonymous variants and 1 frameshift deletion. Sanger sequencing of these remaining 4 variants in all individuals in family A left 2 remaining candidate variants in $N E M F$ (p.H962Y) and SLFN14 (p.V220D) that segregated with disease (Supplemental Table 1; supplemental material available online with this article; doi:10.1172/JCI80347DS1). The exomes of 35 index patients with thrombocytopenia and/or secretion defects who had been recruited to the UK GAPP study were scrutinized for novel variants in either the NEMF or SLFN14 genes. Two further heterozygous missense variants were identified in SLFN14 in affected members of family B (p.K219N) and family C (p.K218E) (Table 1 and Figure 1, A and B). All 3 missense mutations were predicted to result in substitutions in consecutive amino acids within the ATPase-AAA-4 domain of the protein encoded by SLFN14 (Figure 1B). These 3 SLFN14 variants are not present in the Exome Aggregation Consortium data set of 61,486 unrelated individuals, sequenced as part of various disease-specific and 
A

Family A

Family B

Family C
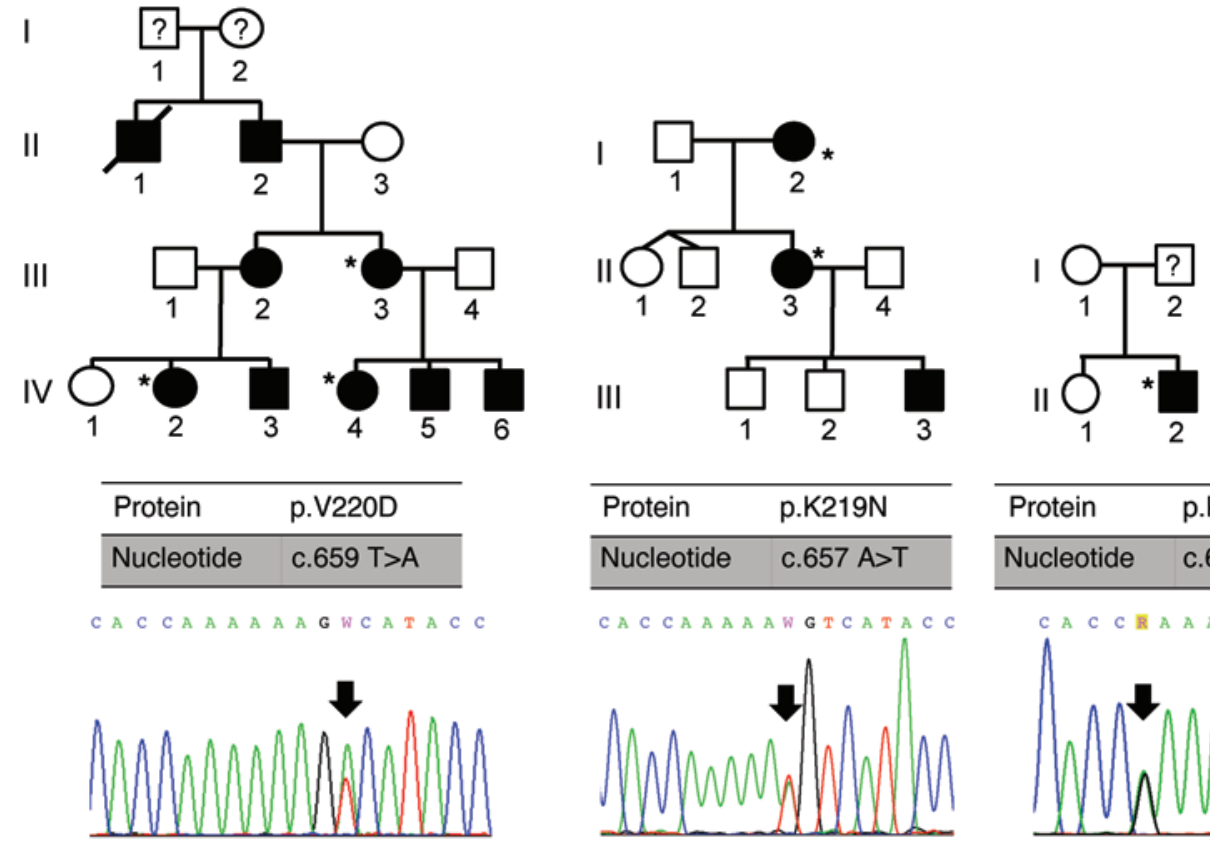

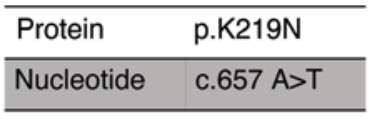

C A C C A A A A W G TCA T A C C

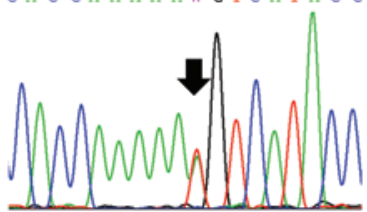

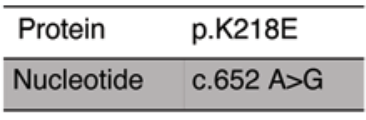

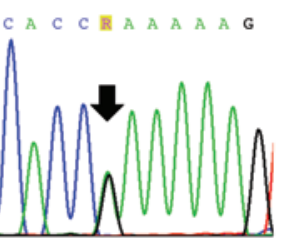

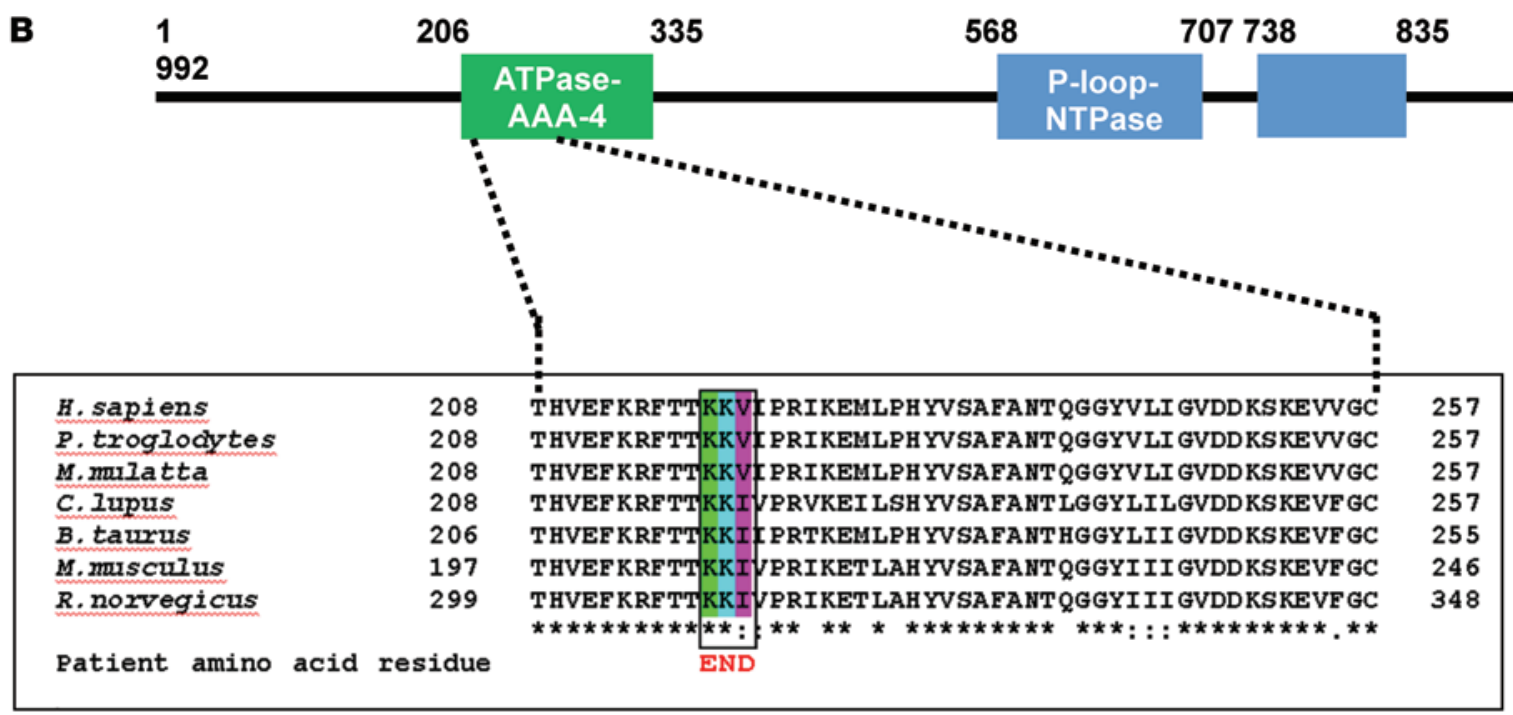

Figure 1. Identification of SLFN14 mutations in 3 unrelated families with a dominant form of thrombocytopenia. (A) Pedigrees from 3 families with moderate thrombocytopenia. Affected individuals are shaded; question marks denote that platelet count is unknown; asterisks indicate those patients whose exomes were sequenced; and arrows indicate nucleotide change. Representative Sanger electropherograms confirming SLFN14 mutations in patients are shown below the relevant families. (B) Linear domain organization of SLFN14 protein, showing the amino acid position of the 3 different missense SLFN14 mutations (K218E, K219N, and V220D) located in the ATPase-AAA-4 domain and conservation of the protein in higher order species.

population genetic studies (http://exac.broadinstitute.org/gene/ ENSG00000236320) and the latest version of dbSNP build 138.

Affected members of families $\mathrm{B}$ and $\mathrm{C}$ had remarkably similar platelet counts and platelet function defects. Family B included 3 patients, of which 2 were recruited to the study (I:2 and II:3). The proband in family B (II:3) was 35 years old when recruited to the study with a platelet count of $68 \times 10^{9} / 1$ and a history of spontaneous epistaxis starting in childhood. Her mother (I:2) had a platelet count in whole blood of $83 \times 10^{9} / 1$, with a less severe bleeding history. The platelet count in patient
II:3 was too low for lumiaggregometry (8), and so platelet function was analyzed by flow cytometry for P-selectin expression, revealing reduced responses to collagen-related peptide and PAR-1 peptide (Table 1). In line with this, lumiaggregometry on patient I:2 revealed deaggregation to ADP, collagen, and PAR-1 and reduced ATP secretion (Table 1). These results are similar to those seen in affected members of family A (Figure 2, A and B).

The index case in family C (II:2) was 3 years old at the time of enrollment. His platelet count in whole blood was $89 \times 10^{9} / 1$, and he was noted to bruise easily from a young age. Lumiag- 
A $\quad \operatorname{PAR}-1(100 \mu \mathrm{M})$

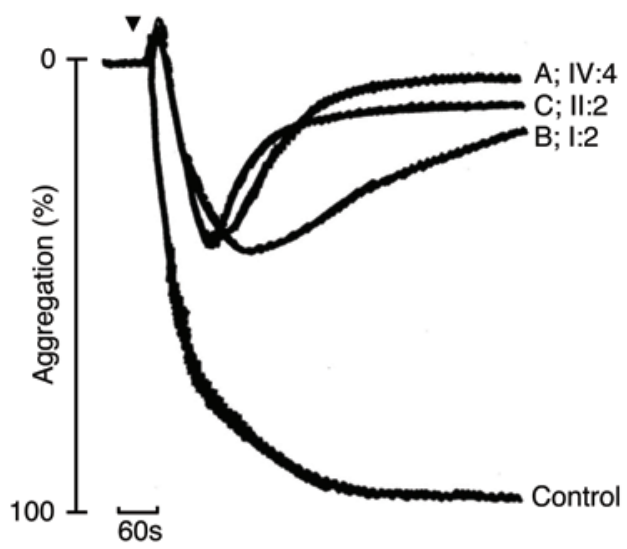

B

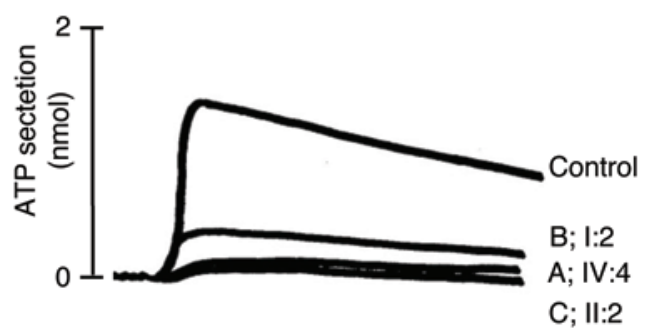

$\operatorname{ADP}(10 \mu \mathrm{M})$

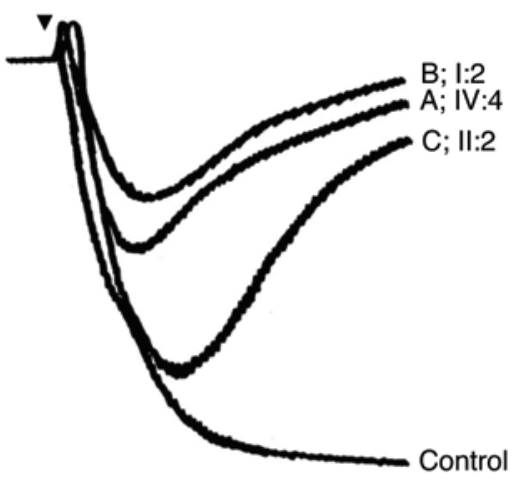

Collagen $(3 \mu \mathrm{g} / \mathrm{ml})$

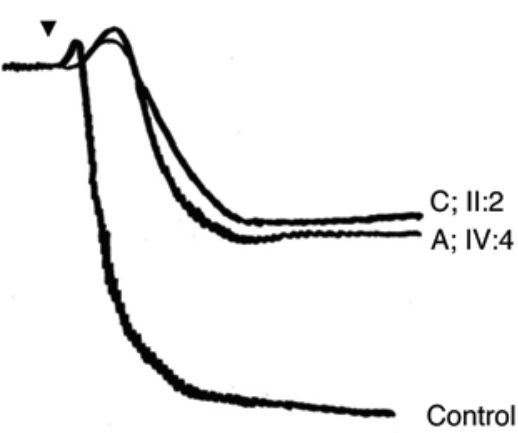

$\mathrm{P}$ selectin (CD62P) response

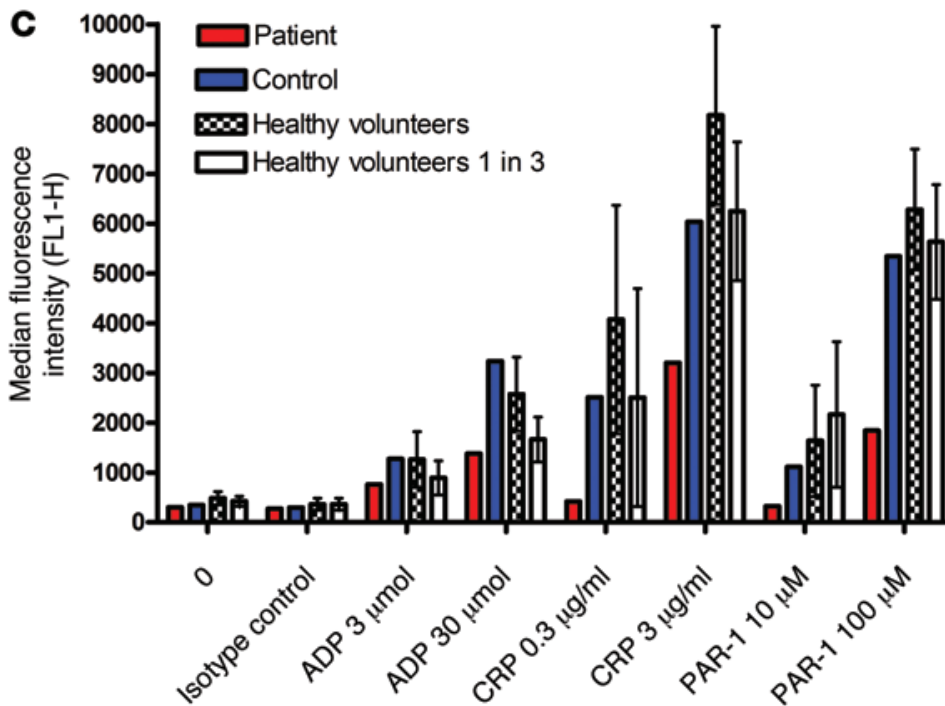

Figure 2. Platelet phenotyping of patients from the 3 families with SLFN14 mutations. (A) Representative impaired aggregation traces in plateletrich plasma from patients from the 3 families (family A, IV:4; family B, I:2; family C, II:2; and representative control), following lumiaggregometry performed on undiluted platelet-rich plasma to assess the percentage aggregation after stimulation with ADP (10 $\mu \mathrm{M})$, collagen ( $3 \mu \mathrm{g} / \mathrm{ml})$, and PAR-1 peptide $(100 \mu \mathrm{M})$. (B) Reduced ATP secretion from dense granules in representative patients from each of the 3 families using Chronolume after stimulation with PAR-1 peptide $(100 \mu \mathrm{M})$. Lumiaggregometry studies shown in $\mathbf{A}$ and $\mathbf{B}$ were repeated once and performed on single platelet samples from multiple affected individuals (family $A, n=5$; family $B, n=1$; and family $C, n=1$ ). (C) Flow cytometric assessment of platelet function in platelet-rich plasma from patient III.2 from family A ( $n=1$ from one experiment). Agonist responses are determined using anti-CD62P. IgCk 1 , isotype control. Data for healthy volunteers are shown as mean \pm SD $(n=9)$. Data for healthy volunteers (1:3 dilution) (with PBS) are shown to demonstrate the effect of moderate thrombocytopenia alone on this assay.

gregometry findings in this patient are similar to those of study participants from families $\mathrm{A}$ and $\mathrm{B}$, with a selective loss of response to ADP, collagen, and PAR-1 and normal responses to arachidonic acid (Table 1, Figure 2, and Supplemental Figure 1).

In humans and mice, SLFN14 is located in a SLFN cluster with other schlafen paralogs (9). Members of the SLFN family are highly conserved among mammalian species. SLFN family proteins contain a unique motif of unknown function, the "SLFN box," and an AAA domain. The $\mathrm{AAA}^{+}$domain consists of a P-loop NTPase implicated in ATP/GTP binding and hydrolysis (10). The SLFN family members are divided into 3 groups. SLFN5, SLFN8, SLFN9, SLFN10, and SLFN14 all belong to group 3, although SLFN14 is unique in containing a putative nuclear localization RKRRR motif in its C-terminus extension (10). The SLFN family of proteins have been suggested to be critical for a variety of processes, including cell-cycle regulation, proliferation, and differentiation (10-14). Recently, data have been published suggesting an important function for SLFN14 as an endoribonuclease, regulating rRNA and ribosome-associated mRNA cleavage and translational control in rabbit reticulocytes (15).

Rowley et al. (16) previously described SLFN14 mRNA expression in human and murine platelets. We identified expression of SLFN14 mRNA and protein in immature and mature megakaryocytes derived from $\mathrm{CD} 34^{+}$hematopoietic progenitor cells isolated from cord blood (Supplemental Figure 2). Expression of SLFN14 protein was confirmed by Western blotting of platelets from 11 healthy individuals, normalized to GAPDH loading controls (Supplemental Figure 3). Levels of SLFN14 in healthy volunteers ranged from 1.0 to 2.3 arbitrary units (mean $1.5,95 \%$ CI $1.2-1.8)$. 

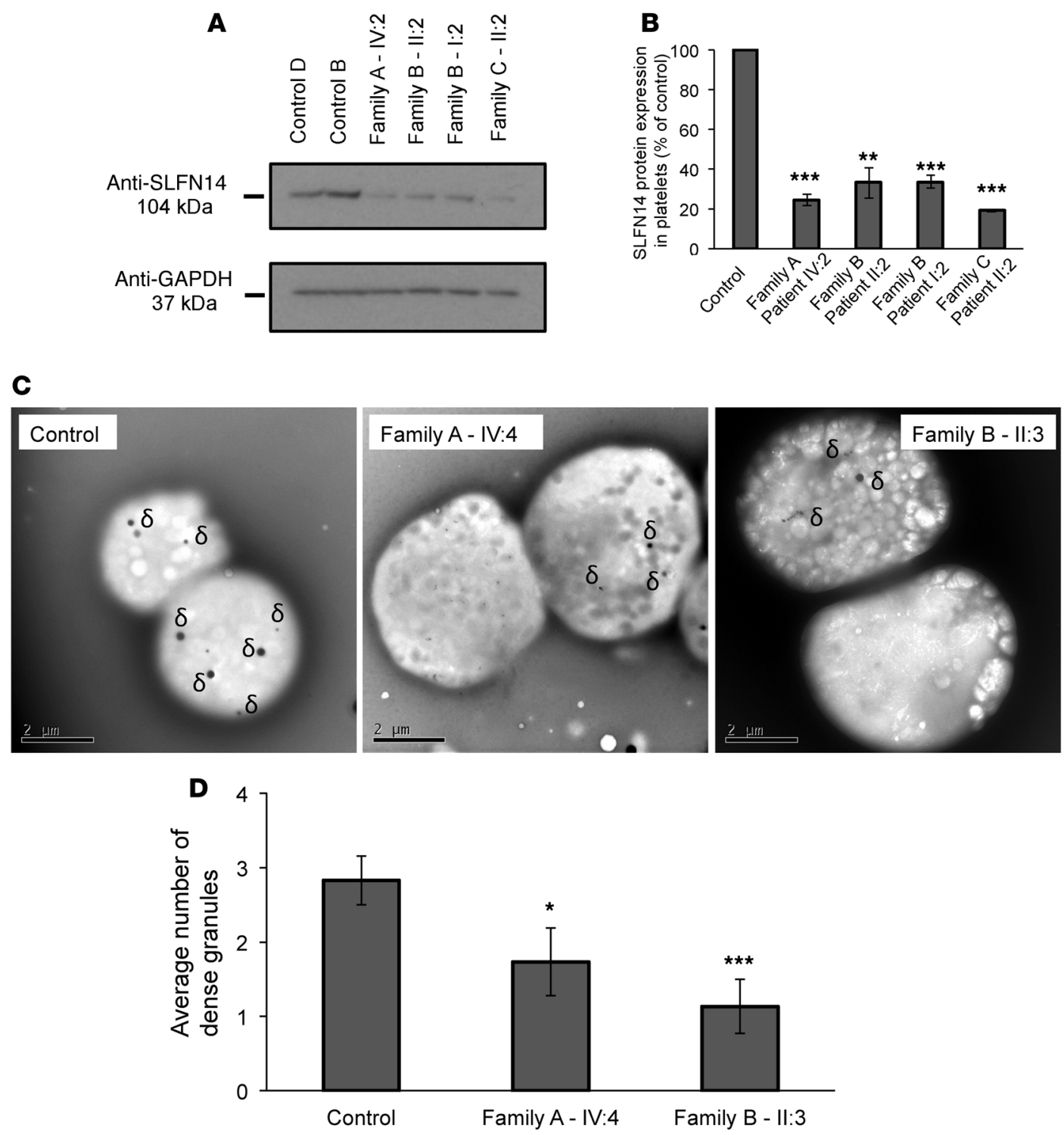

Figure 3. Functional characterization of SLFN14 in patient platelets. (A) Representative Western blot analysis of healthy volunteer and patient platelet lysates, demonstrating decreased SLFN14 protein levels in patient platelets when compared with healthy individuals. (B) Densitometry quantification of 4 independent Western blots (A) performed using a single platelet sample obtained from each patient or healthy volunteer. ${ }^{* *} P \leq 0.005,{ }^{* * *} P \leq 0.001$, compared with control, Student's $t$ test. (C) Whole-mount electron microscopy images showing reduced dense granules $(\delta)$ in patient platelets in comparison to healthy volunteer platelets. Scale bar: $2 \mu \mathrm{m}$.(D) Quantification of data in $\mathbf{C} .{ }^{*} P \leq 0.05,{ }^{* * *} P \leq 0.001$, compared with control, Student's $t$ test. 40 platelets were analyzed per patient/healthy volunteer from a single platelet sample. All values are mean \pm SEM.

The effect of SLFN14 variants on protein expression in platelets from affected family members was investigated by Western blotting (Figure 3A). Platelet lysates from carriers of the 3 SLFN14 variants showed a 65\%-80\% reduction in SLFN14 protein expression when compared with that of control platelets (Figure 3, A and B). Patient III:2 from family A expressing the p.V220D mutation demonstrated a reduction in SLFN14 expression to approximately $24 \%$ of that of control $(P \leq 0.001)$; patients I:2 and II:3 from family B expressing the K219N mutation showed a reduction to $33 \%(P \leq 0.005)$ and $34 \%$ $(P \leq 0.001)$ of that of control, respectively; and patient II:2 from family C expressing the $\mathrm{K} 218 \mathrm{E}$ mutation showed a decrease to $19 \%$ of that of control $(P \leq 0.001)$. This reduction in SLFN14 levels in all 3 families is over $50 \%$ despite the heterozygosity, suggesting that the mutant gene/protein influences stability of the wild-type protein. Importantly, the reduction in SLFN14 levels in platelets from carriers of the SLFN14 variants was confirmed in overexpression studies, in which, despite unaltered transfection efficiency, average field-ofview intensity measurements, and Western blot analysis demonstrated a significant reduction in expression of all SLFN14 variant constructs compared with the that of the wild-type construct, i.e., SLFN14(K218E)-myc, SLFN14(K219N)-myc, and SLFN14(V22OD)-myc expression was reduced to $5 \%, 8 \%$, and $52 \%$ of SLFN14(WT)-myc expression, respectively (Supplemental Figure 4). The much larger reduction in both SLFN14(K218E)-myc and SLFN14(K219N)-myc expression may be a result of increased instability in these mutations in comparison to the SLFN14(V22OD)-myc mutant. 
Expression of SLFN14(WT)-myc in HEK293T cells revealed a punctate structure localized throughout the cytoplasm, with low level nuclear punctate staining also observed. No significant difference in protein localization was observed between overexpression of all SLFN14 mutants and the wild-type construct (Supplemental Figure 5).

The morphology of platelets from patients carrying heterozygous SLFN14 mutations was examined by transmission electron microscopy. Compared with platelets from healthy volunteers, no significant difference in $\alpha$ granule number or platelet ultrastructure was observed (Supplemental Figure 6). A small increase in platelet area was also observed corresponding with data shown in Table 1; however, a statistically significant increase was observed only in patient I:2 of family B $(P \leq 0.005)$. (Supplemental Figure 6).

Whole-mount electron microscopy was used to quantify dense granules numbers within affected patient and healthy volunteers platelets, and a significant reduction in dense granule number was observed in patient platelets from families A and B, patients IV:4 and II:3, respectively (Figure 3, C and D). This observation correlates with the reduced ATP secretion measured by lumiaggregometry (Figure 2). Spreading of platelets on fibrinogen was not significantly different in platelets between affected individuals and healthy controls (Supplemental Figure 7).

In summary, we report 3 unrelated families with a dominantly inherited moderate thrombocytopenia, with a disproportionate bleeding phenotype. A combination of extensive platelet phenotyping and WES identified 3 mutations in SLFN14, which underlies a moderate thrombocytopenia with platelet secretion defects. The affected patients have a distinct platelet phenotype with decreased responses to ADP, collagen, and PAR-1, pointing toward a defect in the $G_{i}$ signalling pathway. Reduced dense granule secretion may also result in a loss of positive feedback during platelet activation. Platelets from affected patients displayed a relatively normal ultrastructure but show a significant reduction in dense granule number, correlating with lumiaggregometry data showing decreased ATP secretion, and provide a basis for future mechanistic studies. This is the first description to our knowledge of a major role for SLFN14 in human disease.

\section{Methods}

Further methods are detailed in the Supplemental Methods.

Statistics. In studies in which statistical analyses were performed, a 2-tailed Student's $t$ test was used to generate $P$ values. $P$ values less than or equal to 0.05 were considered significant.

Study approval. The GAPP study was approved by the National Research Ethics Service Committee West Midlands - Edgbaston (REC reference 06/MRE07/36), and participants gave written informed consent in accordance with the Declaration of Helsinki. This study is registered at ISRCTN (http://www.isrctn.org) as ISRCTN77951167. The GAPP study is included in the National Institute for Health Research Non-Malignant Haematology study portfolio (ID 9858).

\section{Acknowledgments}

We thank the families for providing samples and our clinical and laboratory colleagues for their help. This work was supported by the British Heart Foundation (RG/ PG/13/36/30275; RG/09/007; FS/13/70/30521), an MRC Doctoral Training grant (B. Johnson), a Wellcome Trust Combined Training Programme Fellowship (093994) (to G.C. Lowe), and the Platelet Charity (formerly known as FunnyBlood). The authors also acknowledge support from the Department of Health via the National Institute for Health Research (NIHR). We thank the NIHR Non-Malignant Haematology Specialty Group for their help in recruiting to the study and all our clinical investigators and collaborators. See the Supplemental Acknowledgments for study group details.

Address correspondence to: Neil V. Morgan, Centre for Cardiovascular Sciences, University of Birmingham, Institute of Biomedical Research, Edgbaston, Birmingham, B15 2TT, United Kingdom. Phone: 44.121.414.6820; E-mail: n.v.morgan@bham.ac.uk.
1. Nurden AT, Nurden P. Inherited thrombocytopenias. Haematologica. 2007;92(9):1158-1164.

2. Noris P, et al. Application of a diagnostic algorithm for inherited thrombocytopenias to 46 consecutive patients. Haematologica. 2004;89(10):1219-1225.

3. Balduini CL, Pecci A, Noris P. Inherited thrombocytopenias: the evolving spectrum. Hamostaseologie. 2012;32(4):259-270.

4. Watson SP, Lowe GC, Lordkipanidze M, Morgan NV. Genotyping and phenotyping of platelet function disorders. J Thromb Haemost. 2013;11(suppl 1):351-363.

5. Jones S, et al. Exomic sequencing identifies PALB2 as a pancreatic cancer susceptibility gene. Science. 2009;324(5924):217.

6. Mardis ER, et al. Recurring mutations found by sequencing an acute myeloid leukemia genome. NEngl JMed. 2009;361(11):1058-1066.

7. Ng SB, et al. Targeted capture and massively parallel sequencing of 12 human exomes. Nature. 2009;461(7261):272-276.
8. Dawood BB, et al. Evaluation of participants with suspected heritable platelet function disorders including recommendation and validation of a streamlined agonist panel. Blood. 2012;120(25):5041-5049.

9. Bustos O, et al. Evolution of the Schlafen genes, a gene family associated with embryonic lethality, meiotic drive, immune processes and orthopoxvirus virulence. Gene. 2009;447(1):1-11.

10. Geserick P, Kaiser F, Klemm U, Kaufmann $\mathrm{SH}$, Zerrahn J. Modulation of T cell development and activation by novel members of the Schlafen (slfn) gene family harbouring an RNA helicase-like motif. Int Immunol. 2004;16(10):1535-1548.

11. Brady G, Boggan L, Bowie A, O’Neill LA. Schlafen- 1 causes a cell cycle arrest by inhibiting induction of cyclin D1. J Biol Chem. 2005;280(35):30723-30734.

12. Kuang CY, Yang TH, Zhang Y, Zhang L, Wu Q. Schlafen 1 inhibits the proliferation and tube formation of endothelial progenitor cells. PLoS One. 2014;9(10):e109711.

13. Schwarz DA, Katayama CD, Hedrick SM. Schlafen, a new family of growth regulatory genes that affect thymocyte development. Immunity. 1998;9(5):657-668.

14. van Zuylen WJ, et al. Macrophage activation and differentiation signals regulate schlafen- 4 gene expression: evidence for Schlafen- 4 as a modulator of myelopoiesis. PLoS One. 2011;6(1):e15723.

15. Pisareva VP, Muslimov IA, Tcherepanov A, Pisarev AV. Characterization of novel ribosome-associated endoribonuclease SLFN14 from rabbit reticulocytes. Biochemistry. 2015;54(21):3286-3301.

16. Rowley JW, et al. Genome-wide RNA-seq analysis of human and mouse platelet transcriptomes. Blood. 2011;118(14):e101-e111.

17. Lowe GC, Lordkipanidze M, Watson SP. Utility of the ISTH bleeding assessment tool in predicting platelet defects in participants with suspected inherited platelet function disorders. J Thromb Haemost. 2013;11(9):1663-1668. 